\title{
Co-infections with Babesia microti and Plasmodium parasites along the China-Myanmar border
}

\author{
Xia Zhou ${ }^{1,2}$, Sheng-Guo Li ${ }^{3}$, Shen-Bo Chen', Jia-Zhi Wang ${ }^{3}$, Bin Xu' ${ }^{1}$, He-Jun Zhou', Hong-Xiang Zhu Ge², \\ Jun-Hu Chen ${ }^{1}$ and Wei Hu ${ }^{1,4^{*}}$
}

\begin{abstract}
Background: Babesiosis is an emerging health risk in several parts of the world. However, little is known about the prevalence of Babesia in malaria-endemic countries. The area along the China-Myanmar border in Yunnan is a main endemic area of malaria in P.R. China, however, human infection with Babesia microti (B. microti) is not recognized in this region, and its profile of co-infection is not yet clear.

Methods: To understand its profile of co-infections with B. microti, our investigation was undertaken in the malaria-endemic area along the China-Myanmar border in Yunnan between April 2012 and June 2013. Four parasite species, including B. microti, Plasmodium falciparum (P. falciparum), P. vivax, and P. malariae, were identified among 449 suspected febrile persons detected by nested polymerase chain reaction (PCR) assay based on small subunit ribosomal ribonucleic acid (RNA) genes of B. microti and Plasmodium spp.

Results: Of all the collected samples from febrile patients, mono-infection with B. microti, P. vivax, P. falciparum, and $P$. malariae accounted for 1.8\% (8/449), 9.8\% (44/449), $2.9 \%$ (13/449), and $0.2 \%$ (1/449), respectively. The rate of mixed infections of $B$. microti with $P$. falciparum or $P$. vivax are both $0.2 \%(1 / 449)$, and mixed infections of $P$. falciparum and $P$. vivax accounted for $1.1 \%(5 / 449)$.

Conclusions: This report supports the hypothesis that babesiosis caused by $B$. microti is emerging along the China-Myanmar border in the Yunnan province, P.R. China, but it was ignored because of low parasitemia or mixed infection with Plasmodium spp. More sensitive and specific diagnosis methods are needed to find the rapid response mechanism of emergency for babesiosis and malaria co-prevalence areas.
\end{abstract}

Keywords: Babesia, Plasmodium, Co-infection, China-Myanmar border

\section{Multilingual abstracts}

Please see Additional file 1 for translations of the abstract into the six official working languages of the United Nations.

\section{Background}

Babesiosis is a typical zoonotic disease which is caused by Babesia spp., tick-borne intraerythrocytic protozoan parasites. The first definitive case of human babesiosis, was documented in a Yugoslavian farmer in 1957 [1]. The

\footnotetext{
* Correspondence: huw@fudan.edu.cn

${ }^{1}$ National Institute of Parasitic Diseases, Chinese Center for Disease Control and Prevention, WHO Collaborating Centre for Malaria, Schistosomiasis and Filariasis, Key Laboratory of Parasite \& Vector Biology Ministry of Health, Shanghai 200025, China

${ }^{4}$ Department of Microbiology and Microbial Engineering, School of Life Science, Fudan University, Shanghai 200433, China

Full list of author information is available at the end of the article
}

first case in an immunocompetent person was identified on Nantucket Island, off the coast of Massachusetts, in 1969 [2]. In fact, the causative agent was Babesia microti, and the vector was the Ixodes dammini tick (now referred to as I. scapularis) [3]. Babesiosis is now classified as a notifiable disease and is recognized as an emerging health risk in several parts of the world [4], but little is known about the its prevalence in malaria-endemic countries, where misidentification as Plasmodium spp. probably occurs (http://dpd.cdc.gov/dpdx/HTML/Babe siosis.htm). In Asia, B. microti-like organisms have caused illness in Japan, Taiwan, and mainland China [5-8]. In Europe, a few human babesiosis cases have been attributed to $B$. divergens, the cattle species transmitted by I. ricinus [9-11]. Hundreds of human babesiosis cases in the northeast and midwest United States have been attributed

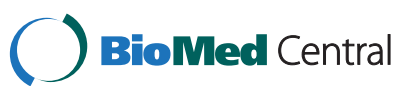


to B. microti [12]. Sporadic cases of babesiosis have been reported in Africa, Australia, and South America [9,13,14].

Recently, co-infection of parasites in humans has been noticed in many areas [15-17]. Human babesiosis has sometimes been diagnosed initially as malaria because of the similarity between the two diseases or the two parasites $[18,19]$. Thus, it is likely that cases of human babesiosis in countries in which malaria is endemic have been overlooked or misdiagnosed as malaria. The area along the China-Myanmar border in Yunnan has been reported as a main endemic area of malaria in the People's Republic of China (P.R. China) [20,21], however $B$. microti has not been recognized as being endemic in this region in the past. Therefore, surveillance was carried out between April 2012 and June 2013 on the presence of $B$. microti from the patients' blood as detected by molecular tools collected in this area.

\section{Methods}

\section{Study area and population}

The area along the China-Myanmar border in Yunnan is a main endemic area of malaria in China. The region is hilly and covered largely by primary and secondary rainforests. Inhabitants of the division are mainly rural indigenous people. Besides working for the logging industry in the surrounding jungles, inhabitants of the Tengchong county in the Yunnan province are actively involved in mining, farming, hunting, logging, and working abroad in Myanmar but coming back frequently. The population of the Tengchong county is served by a general hospital, a polyclinic at the county's Center for Disease Control and Prevention (CDC), and 15 government health clinics.

The study was reviewed and approved by the Ethics Committee at the National Institute of Parasitic Diseases, Chinese Center for Disease Control and Prevention. Four hundred and forty-nine blood samples in people with malaria-like symptoms were obtained between April 2012 and June 2013 in the area along the China-Myanmar border in the Yunnan province of China. Written consent was obtained from each person before blood samples were obtained.

\section{Blood samples}

This survey was carried out on the population seeking care at the Tengchong county CDC. During the study period, finger-prick blood samples on filter paper from 449 febrile patients with malaria-like symptoms were taken. The thick and thin blood films were stained with Giemsa and examined by staff at the diagnostic laboratories of the polyclinic and hospital.

A drop $(20 \mu \mathrm{L}-50 \mu \mathrm{L})$ of blood was placed directly on a pre-marked filter paper. DNA was extracted from the blood spots with QIAGEN (QIAamp DNA Blood Mini
Kit, Biosearch Technologies, Inc. USA) and all of the positive samples were used for sequencing the small subunit ribosomal RNA (SSU rRNA) of the parasites.

\section{SSU rRNA Sequence and phylogenetic analysis}

After the screening of Plasmodium spp. infection by microscopy, all the samples' DNA templates for a nested polymerase chain reaction (PCR) were prepared from whole blood spots on the filter paper. Two microliters of PCR product from each, amplified by Plasmodium genus-specific primer pair rPLU1 and rPLU5, were subjected to a second PCR amplification with speciesspecific primer pairs rVIV1 and rVIV2 for $P$. vivax, rFAL1 and rFAL2 for $P$. falciparum, rMAL1 and rMAL2 for $P$. malariae, and rOVA1 and rOVA2 for P. ovale [22]. B. microti-specific fragments of SSU rRNA were amplified from the DNA extracted from all the febrile patients by nested PCR with two sets of B. microti-specific primers, Bab1-Bab4, and Bab2-Bab3 (B. microti detection PCR), in accordance with the method established by Persing et al. [23]. For B. microti detection PCR-positive samples, a more accurate analysis by amplification of the full-sized sequence $(\sim 1.7 \mathrm{~kb})$ encoding the $18 \mathrm{~S}$ rRNA gene of the Babesia parasite from the patients was applied with the nested PCR primers to confirm the infections. The first round primers were Piro1F: 5'-CCATGCATGTCTWAGTAYAARCTTTTA-3' and rRNA-3': 5'-ATCCTTCYGCAGGTTCACCTAC-3'. The second round primers were BablA: 5'-GTCTTAG TATAAGCTTTTATACAGCG-3' and Prio6R: 5'-CTCCT TCCTY TAAGTGATAAGGTTCAC-3', as described by Medlin et al. [24,25]. PCR amplification for each sample was done in a $50 \mu \mathrm{L}$ reaction mixture containing $400 \mathrm{mM}$ each of deoxynucleoside triphosphates, $0.25 \mathrm{mM}$ of primer, ten to $100 \mathrm{ng}$ of template DNA, and $2.5 \mathrm{U}$ of Taq DNA polymerase (TIANGEN Biochemical) in $50 \mathrm{ml}$ of the PCR buffer supplied together with the enzyme. PCR amplification parameters were: $94^{\circ} \mathrm{C}$ for four minutes, 35 cycles at $94^{\circ} \mathrm{C}$ for 30 seconds, $55^{\circ} \mathrm{C}$ for one minute and $72^{\circ} \mathrm{C}$ for $2 \mathrm{~min}$, followed by a final extension for ten minutes at $72^{\circ} \mathrm{C}$. PCR products amplified with nested primers were analyzed by agarose gelelectrophoresis. DNA bands were removed from the gel, purified by using the QIAquick Gel Extraction Kit (QIAGEN, Valencia, CA, USA), and ligated to T-cloning vector (Invitrogen, Carlsbad, CA, USA) according to protocols provided by the manufacturers.

To avoid errors caused by contamination of PCR products, DNA was extracted and divided into several aliquots in a safety cabinet placed in a room in which PCR products had never been treated. Detection and confirmation of PCR assays were separately carried out with the different aliquots. 
Table 1 B. microti and Plasmodium spp. detection in 449 suspected febrile patients in the area along the China-Myanmar border in the Yunnan province between April 2012 and June 2013

\begin{tabular}{lr}
\hline Parasite & No. (\%) persons \\
\hline B. microti & $8(1.8)$ \\
B. microti / P. vivax & $1(0.2)$ \\
B. microti / P. falciparum & $1(0.2)$ \\
P. vivax & $44(9.8)$ \\
P. falciparum & $13(2.9)$ \\
P. vivax / P. falciparum & $5(1.1)$ \\
P. malariae & $1(0.2)$ \\
\hline
\end{tabular}

Plasmid inserts were then sequenced. Sequence identity was confirmed by random basic local alignment search tool analysis of sequences in GenBank (http://blast. ncbi.nlm.nih.gov/). Phylogenetic relationships of unique sequences amplified were constructed by using the neighbor-joining method using MegAlign software (DNASTAR, Inc. Madison, WI, USA).

\section{Results}

Detection of B. microti and Plasmodium spp.

Four species of parasites taking residence in human erythrocyte (B. microti, $P$. falciparum, $P$. vivax, and $P$. malariae) were identified in 449 suspected febrile persons. Mono-infection with B. microti, P. vivax, P. falciparum, or P. malariae accounted for $1.8 \%$ (8/449), $9.8 \%$ (44/449), $2.9 \%$ (13/449), and $0.2 \%$ (1/449), respectively, of the total infections. The rate of mixed infections of $B$. microti and P. falciparum or $P$. vivax were both $0.2 \%(1 / 449)$, and mixed infections of $P$. falciparum and $P$. vivax accounted for $1.1 \%(5 / 449)$ (see Table 1 and Figure 1). The total prevalence of $B$. microti was
2.2\% (10/449). One P. falciparum mono-infection case, five $P$. vivax, and eight $B$. microti cases were detected from microscopy screening negative samples. Two mixed infection cases of B. microti co-infection with P. falciparum or $P$. vivax were initially diagnosed as mono-infections of Plasmodium spp. by microscopy screening.

\section{SSU rRNA sequence and phylogenetic analysis}

All the positive samples were sequenced. Sequence identity was confirmed by random basic local alignment search tool analysis of sequences in GenBank (http:// blast.ncbi.nlm.nih.gov/). Novel sequences were deposited in GenBank with accession nos. KF410824-KF410827. Phylogenetic relationships of unique sequences amplified by using nested primers with corresponding reference sequences were constructed by using the neighborjoining method using MegAlign software (DNASTAR, Inc. Madison, WI, USA). All sequences clustered with reference sequences of $B$. microti were from different countries or regions, which suggested that all sequences were species specific. Phylogenetic analysis showed that amplified products were species specific (see Figure 2).

\section{Morphological characteristics}

We observed the intro-erythrocytic cycle of the field samples in Giemsa-stained blood films from individuals that were identified by PCR as having B. microti infections. Babesia species resemble P. falciparum, however, Babesia spp. has several distinguishing features: the early trophozoite stage of $B$. microti is smaller than that of $P$. falciparum and the infected erythrocytes are without any pigments. And the late stage of trophozoites is pleomorphic and can be vacuolated. Ring forms of B. microti appeared smaller than that of P. falciparum and do not produce pigment (see Figure 3A), while early

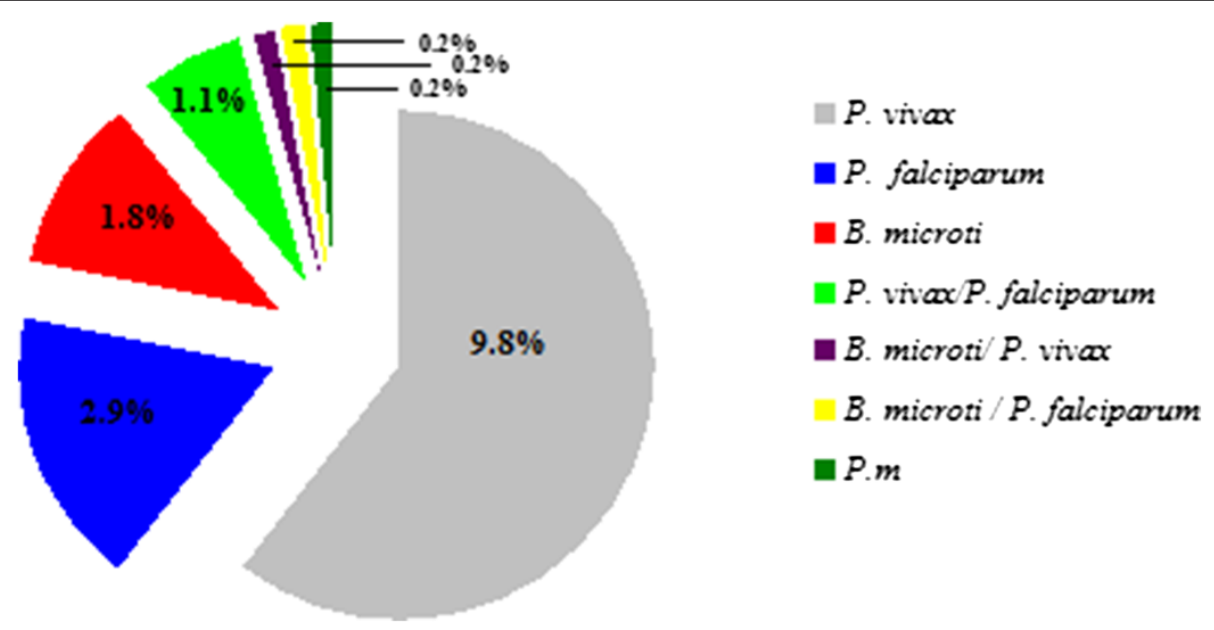

Figure 1 Infection rate of $B$. microti and Plasmodium spp. in 449 suspected febrile patients in the area along the China-Myanmar border in the Yunnan province between April 2012 and June 2013. 


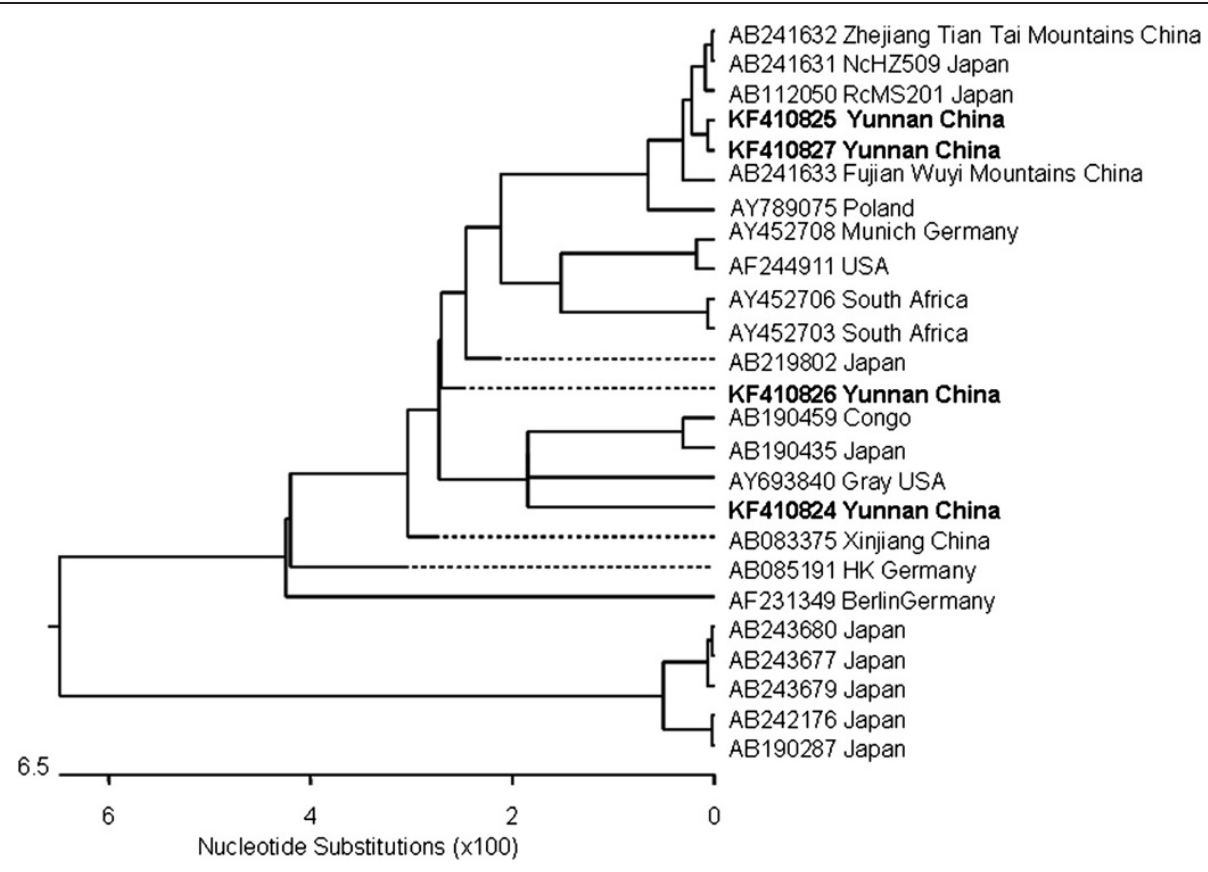

Figure 2 Phylogenetic analysis of $18 \mathrm{~S}$ small subunit SSU rRNA gene sequences of $B$. microti in the malaria-endemic area along the China-Myanmar border in the Yunnan province, April 2012 to June 2013. Fragments of 18S SSU rRNA gene sequences of samples were analyzed by aligning with published homologous sequences of $B$. microti from different countries or regions. A phylogenetic tree was constructed on the basis of similarities by the MegAlign software (DNASTAR, Inc. Madison, WI, USA). Novel sequences identified in this study are indicated in boldface. Scale bar indicates nucleotide substitutions per site.
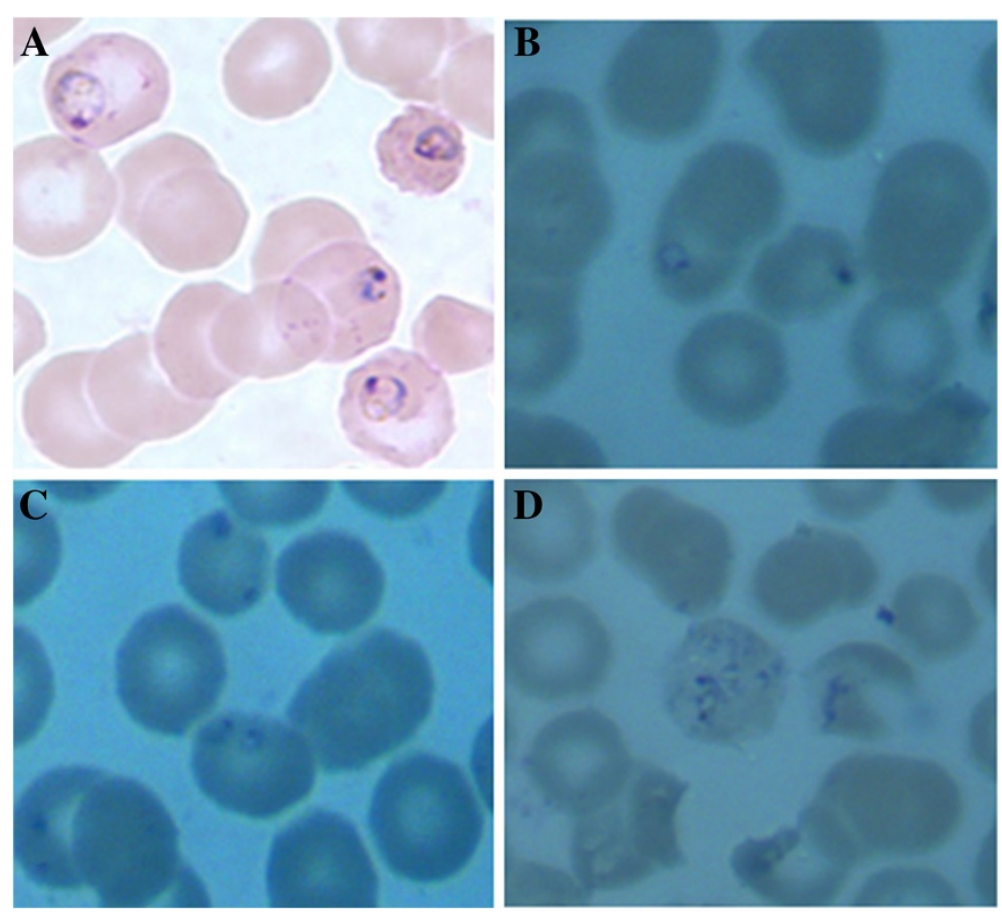

Figure 3 Typical morphology of erythrocytic stages of $B$. microti in patients in the area along the China-Myanmar border in the Yunnan province, P.R. China. Giemsa-stained thin blood films from field samples. A: Early trophozoite or ring form of $P$. falciparum and malaria pigment was scattered in the infected erythrocytes. B: Ring forms of B. microti in one erythrocyte without pigment. C: Two ring forms take residents in one erythrocyte $\mathbf{D}$ : Tetrads of merozoites that are arranged in a cross-like pattern (a so-called Maltese cross). 

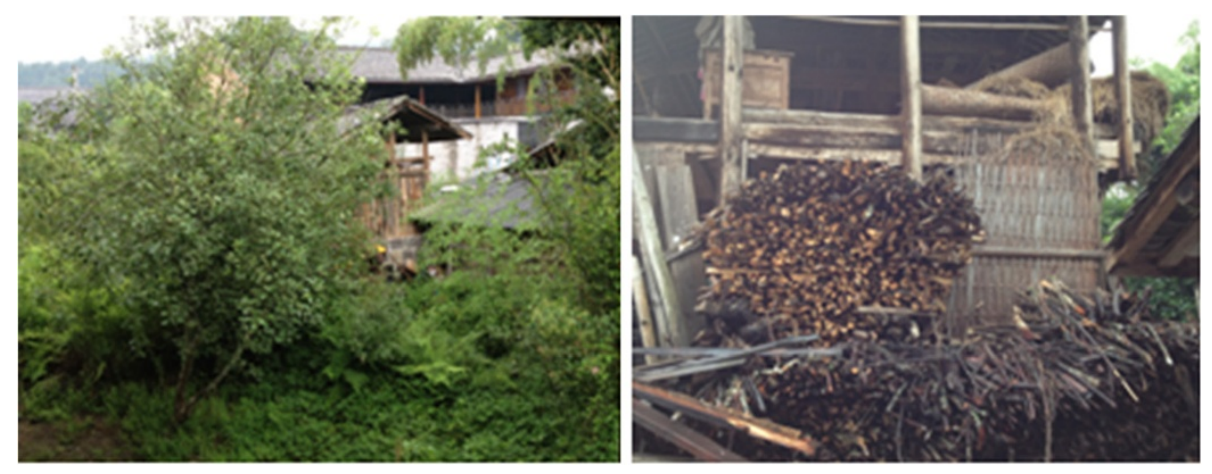

Figure 4 Area along the China-Myanmar border in the Yunnan province, P.R. China (which is hilly, and largely covered by primary and secondary rainforests) with babesiosis and malaria.

trophozoites of $P$. falciparum were larger and malaria pigment was scattered in the infected erythrocytes (see Figure 3B). Occasionally, two trophozoites or ring forms would take residence in one erythrocyte (see Figure 3C). Tetrads of merozoites that are arranged in a cross-like pattern (a so-called Maltese cross) being pathognomonic for babesiosis can be seen (see Figure 3D).

\section{Epidemiological data, clinical history}

The demographic details of ten babesiosis patients detected by nested PCR from 449 individuals were recalled. All of these individuals were from the aforementioned study area (see Figure 4). The brief hospital records and data for clinical presentation, and whether outbound history was available, are shown in Table 2, but the treatment and outcome was not available. All of the patients were adults and complained of fever with chills and rigor before admission to hospital. We tracked two babesiosis cases as well and found that both of them recalled multiple tick bites in the recent past and also participated in outdoor activities in the mountains. One patient had even received blood

Table 2 Demographic and clinical data for patients of babesiosis at dates of admission, in the area along the China-Myanmar border in the Yunnan province, April 2012 to June 2013

\begin{tabular}{lr}
\hline Demographic data $(\mathbf{n}=\mathbf{1 0})$ & \\
\hline Age (years) & \\
Male gender & $10(100 \%)$ \\
Adults (>18 years) & \\
Clinical history and presentations $(\mathrm{n}=10)$ & \\
Temperature at admission $\left({ }^{\circ} \mathrm{C}\right)$ & $37.8(0.82,36.0-38.8)$ \\
Outbound history & $6(60 \%)$ \\
Duration of illness before admission (days) & $6.2(9.4,1-32)$ \\
Fever, chill, and rigor & $10(100 \%)$ \\
\hline
\end{tabular}

Data shown as $\mathrm{n}$ (\% of total), or mean (SD; range) unless otherwise stated. transfusion and blood products for treatment of renalmalaria before because of infection of P. falciparum.

\section{Discussion}

Between 1931 and 1944, Hung S.L. et al. [26] reported several human parasitemia in Chongqing, China, on protozoa taking residents in human erythrocytes, which was described as being similar to P. falciparum, but with fundamental differences of smaller ring forms and no pigments, and so it clearly indicated that it was Babesia spp. infection. These reports came 13 years earlier than the first case of human babesiosis was reported in a Yugoslavian farmer in 1957 [1], and strongly suggested that human Babesia spp. infection may have already existed widely in P.R. China. In fact, Yunnan, Inner Mongolia, Taiwan and Zhejiang, and other regions in P.R. China also have sporadic human babesiosis cases reported from 1984 to 2012 [5,8,27-30]. Sun Y et al. [31] reported that a $B$. microti-like rodent parasite was isolated from the tick, I. persulcatus, collected from the northern forest area of Heilongjiang province, P.R. China. Saito-Ito et al. [32] confirmed Babesia spp. infection of the Kobe strain presented in rodents in Zhejiang, Fujian, and Taiwan. But there are no reports of regional popularity on human babesiosis especilly in these natural foci areas in which Babesia spp. existed in the ticks or/and other reservoir hosts. Our study supports the hypothesis that human babesiosis caused by $B$. microti infection is emerging in areas along the China-Myanmar border in counties such as Tengchong in the Yunnan province, P.R. China, but can be ignored because of mix infection with Plasmodium spp. or low parasitemia.

Both Plasmodium and Babesia species are intraerythrocytic protozoans and they elicit similar inflammatory responses and clinical manifestations that differ markedly in severity. Babesia spp. co-infection with P. falciparum could not be ruled out in one case. The possibility of co-infection with babesial and malarial agents was well described in an infant from the Ivory Coast who was 
quite ill with parasitaemia of 35\% [33]. Co-infection with both agents was reported in another rhesus monkey imported from Guangxi, P.R. China and the suspected infection of $B$. microti-like originated from primatebreeding facility [34]. Here, we detected two cases of febrile patients who were co-infected with Plasmodium spp. and Babesia spp. in malaria endemic area by molecular survey - it was unclear what the dominant parasite was and the microscopy detection of the parasite species of co-infections cases seem more difficult. It was reported that a rhesus monkey that was chronically infected with $B$. microti was able to control infection with P. cynomolgi better than naïve monkeys [35]. The suppression of Plasmodium spp. infection after chronic exposure to $B$. microti also warrants further investigation of a possible protective role of Babesia spp. infection on Plasmodium spp.

The new finding of mono-infection of Babesia and co-infection of Babesia spp. and Plasmodium spp. in the malaria-endemic area of the Tengchong county would bring new challenges to the prevention and controlling of these infectious diseases. It must also be noted that most antimalarial drugs, such as chloroquine, mefloquine, and artemisinin, have no effect on babesiosis [36]. Quinine and clindamycin, the former of which is often used for treatment of drug-resistant malaria, are the first-choice drugs against babesiosis [4,37]. Therefore, babesiosis in areas in which malaria is endemic might be misdiagnosed as drug-resistant malaria. From this standpoint, the identification of babesial parasite infection in the Yunnan province, where malaria is endemic, seems to deserve attention, considering that recent Asian cases of human babesiosis emerged in P. R. China and Japan, where B. microti-like parasites had earlier been identified in rodents. The importance of this finding in human coinfection of Babesia spp. and Plasmodium spp. is also reflected in case therapy as treatment of malaria patients depends on correct diagnosis of parasite species [38]. Ring forms of Babesia spp. may resemble those of $P$. falciparum [9]. Artemisinin-based combination therapies are the recommended first-line treatments of falciparum malaria in all countries where malaria transmitted. There are recent concerns that the efficacy of such therapies has declined on the Thailand-Cambodian and Thailand-Myanmar borders, historically sites of emerging antimalarial-drug resistance [39,40]. The morphology of $P$. falciparum and B. microti are similar especially in the ring form stage which is the main intraerythrocyte of both parasites so human Babesiosis may be misdiagnosed as falciparum malaria in these malaria endemic areas. Giving Artemisinin-based combination therapies to these babesiosis patients due to misdiagnosis would undoubtedly increase the pressure of artemisinin resistance in malariatransmitted areas.

\section{Conclusions}

Babesiosis has sometimes been diagnosed initially as malaria because of the similarity between the two diseases, which is the main reason why it is difficult to detect it in co-infections [41]. This report supports the hypothesis that babesiosis caused by $B$. microti is emerging along the China-Myanmar border in the Yunnan province, P.R. China, but it was ignored because of low parasitemia or mixed infection with Plasmodium spp. It's crucial to apply more sensitive and specific diagnosis methods to set up rapid response mechanisms of emergency for the important infectious diseases in babesiosis and malaria syndemic areas, particularly in places which are weak in surveillance and response systems [42].

\section{Additional file}

Additional file 1: Multilingual abstracts in the six official working languages of the United Nations.

Competing interests

The authors declare that they have no competing interests.

\section{Authors' contributions}

$X Z$ conceived the study, collected the data and analyzed it, and drafted the manuscript. SGL and JZW revised the manuscript and provided intellectual input for the interpretation of the findings. SBC, BX, and HJZ conceived the project and provided technical support for data collection and analysis. JHC, HXZG and WH conceived the study and revised the manuscript. All authors read and approved the final manuscript.

\section{Acknowledgment}

The research has been partially supported by the Special Fund for Health Research in the Public Interest China (No. 201202019), Strengthen Action Plan for Shanghai Public Health System Construction 2011-2013 (GW-11) and by the National S \& T Major Program (No. 2012ZX10004-220).

\section{Author details}

${ }^{1}$ National Institute of Parasitic Diseases, Chinese Center for Disease Control and Prevention, WHO Collaborating Centre for Malaria, Schistosomiasis and Filariasis, Key Laboratory of Parasite \& Vector Biology Ministry of Health, Shanghai 200025, China. ${ }^{2}$ Department of Parasitology, Medical College of Soochow University, No.199 Renai Road, Suzhou 215123, China. ${ }^{3}$ Tengchong Center for Disease Control and prevention, Yunnan province 679100, China. ${ }^{4}$ Department of Microbiology and Microbial Engineering, School of Life Science, Fudan University, Shanghai 200433, China.

Received: 1 August 2013 Accepted: 26 September 2013

Published: 3 October 2013

\section{References}

1. Skrabalo Z, Deanovic Z: Piroplasmosis in man; report of a case. Doc Med Geogr Trop 1957, 9:11-16.

2. Western KA, Benson GD, Gleason NN, Healy GR, Schultz MG: Babesiosis in a Massachusetts resident. N Engl J Med 1970, 283:854-856.

3. Spielman A, Clifford CM, Piesman J, Corwin MD: Human babesiosis on Nantucket Island, USA: description of the vector, Ixodes (Ixodes) dammini, n. sp. (Acarina: Ixodidae). J Med Entomol 1979, 15:218-234.

4. Vannier E, Krause PJ: Human babesiosis. N Engl J Med 2012, 366:2397-2407.

5. Shih CM, Liu LP, Chung WC, Ong SJ, Wang CC: Human babesiosis in Taiwan: asymptomatic infection with a Babesia microti-like organism in a Taiwanese woman. J Clin Microbiol 1997, 35:450-454.

6. Shaio MF, Lin PR: A case study of cytokine profiles in acute human babesiosis. Am J Trop Med Hyg 1998, 58:335-337. 
7. Wei Q, Tsuji M, Zamoto A, Kohsaki M, Matsui T, Shiota T, Telford SR 3rd, Ishihara C: Human babesiosis in Japan: isolation of Babesia microti-like parasites from an asymptomatic transfusion donor and from a rodent from an area where babesiosis is endemic. J Clin Microbiol 2001, 39:2178-2183

8. Yao LN, Ruan W, Zeng CY, Li ZH, Zhang X, Lei YL, Lu QY, Che HL: Pathogen identification and clinical diagnosis for one case infected with Babesia. Zhongguo Ji Sheng Chong Xue Yu Ji Sheng Chong Bing Za Zhi 2012, 30:118-121.

9. Hunfeld KP, Hildebrandt A, Gray JS: Babesiosis: recent insights into an ancient disease. Int J Parasitol 2008, 38:1219-1237.

10. Gorenflot A, Moubri K, Precigout E, Carcy B, Schetters TP: Human babesiosis. Ann Trop Med Parasitol 1998, 92:489-501.

11. Hildebrandt A, Hunfeld KP, Baier M, Krumbholz A, Sachse S, Lorenzen T, Kiehntopf M, Fricke HJ, Straube E: First confirmed autochthonous case Of human Babesia microti infection in Europe. Eur J Clin Microbiol Infect Dis 2007, 26:595-601.

12. Vannier E, Gewurz BE, Krause PJ: Human babesiosis. Infect Dis Clin North Am 2008, 22:469-488

13. Kjemtrup AM, Conrad PA: Human babesiosis: an emerging tick-borne disease. Int J Parasitol 2000, 30:1323-1337.

14. Senanayake SN, Paparini A, Latimer M, Andriolo K, Dasilva AJ, Wilson H, Xayavong MV, Collignon PJ, Jeans P, Irwin PJ: First report of human babesiosis in Australia. Med J Aust 2012, 196:350-352.

15. Mazigo HD, Nuwaha F, Wilson S, Kinung'hi SM, Morona D, Waihenya R, Heukelbach J, Dunne DW: Epidemiology and interactions of human immunodeficiency virus - 1 and schistosoma mansoni in sub-saharan africa. Infect Dis Poverty 2013, 2:2.

16. Li XX, Zhou XN: Co-infection of tuberculosis and parasitic diseases in humans: a systematic review. Parasit Vectors 2013, 6:79.

17. Tian LG, Chen JX, Wang TP, Cheng GJ, Steinmann P, Wang FF, Cai YC, Yin XM, Guo J, Zhou L, Zhou XN: Co-infection of HIV and intestinal parasites in rural area of China. Parasit Vectors 2012, 5:36.

18. Homer MJ, Aguilar-Delfin I, Telford SR 3rd, Krause PJ, Persing DH: Babesiosis. Clin Microbiol Rev 2000, 13:451-469.

19. Dantrakool A, Somboon P, Hashimoto T, Saito-Ito A: Identification of a new type of Babesia species in wild rats (Bandicota indica) in Chiang Mai Province, Thailand. J Clin Microbiol 2004, 42:850-854

20. Moore SJ, Min X, Hill N, Jones C, Zaixing Z, Cameron MM: Border malaria in China: knowledge and use of personal protection by minority populations and implications for malaria control: a questionnaire-based survey. BMC Public Health 2008, 8:344.

21. Liu J, Yang B, Cheung WK, Yang G: Malaria transmission modelling: a network perspective. Infect Dis Poverty 2012, 1:11.

22. Snounou G, Viriyakosol S, Zhu XP, Jarra W, Pinheiro L, Do Rosario VE, Thaithong S, Brown KN: High sensitivity of detection of human malaria parasites by the use of nested polymerase chain reaction. Mol Biochem Parasitol 1993, 61:315-320.

23. Persing DH, Mathiesen D, Marshall WF, Telford SR, Spielman A, Thomford JW, Conrad PA: Detection of Babesia microti by polymerase chain reaction. J Clin Microbiol 1992, 30:2097-2103.

24. Medlin L, Elwood HJ, Stickel S, Sogin ML: The characterization of enzymatically amplified eukaryotic 16S-like rRNA-coding regions. Gene 1988, 71:491-499.

25. Saito-Ito A, Tsuji M, Wei Q, He S, Matsui T, Kohsaki M, Arai S, Kamiyama T, Hioki K, Ishihara C: Transfusion-acquired, autochthonous human babesiosis in Japan: isolation of Babesia microti-like parasites with hu-RBC-SCID mice. J Clin Microbiol 2000, 38:4511-4516.

26. SL H: Notes on a species of malaria parasite finding from Bei-Bei. National Medical Journal of China (Chongqing ed) 1944, 19:571-573.

27. Li JFMD, Wang QF, et al: The discovery of human Babesiasis. Chinese Journal of Veterinary Medicine 1984, 6:19-20.

28. Su GGn Zhao NF, Ye YX: A babesia case report. Chinese Journal of Zoonose 2002, 18:112-155.

29. Shi ZBLZ, Gao QR, Ding YM: One case of human babesia infection. Chinese Journal of Par asitology \& Parasitic Diseases 1996, 14:240.

30. HX W: One case of babesiosis in Kunming. International Journal of Medical Parasitic Diseases 2012, 39:190-192.

31. Sun Y, Liu G, Yang L, Xu R, Cao W: Babesia microti-like rodent parasites isolated from Ixodes persulcatus (Acari: Ixodidae) in Heilongjiang Province, China. Vet Parasitol 2008, 156:333-339.
32. Saito-Ito A, Takada N, Ishiguro F, Fujita $H$, Yano $Y$, Ma XH, Chen ER: Detection of Kobe-type Babesia microti associated with Japanese human babesiosis in field rodents in central Taiwan and southeastern mainland China. Parasitology 2008, 135:691-699.

33. Vermeil C, Menut J, Miegeville M, Cruziat J, Julienne F, Morin O, Roger AP, Marjolet M, Bouillard C: Babesiasis, pediatric malaria: does confusion exist in Africa? Bull Soc Pathol Exot Filiales 1983, 76:797-804.

34. Voorberg-vd Wel A, Kocken $\mathrm{CH}$, Zeeman AM, Thomas AW: Detection of new Babesia microti-like parasites in a rhesus monkey (Macaca mulatta) with a suppressed Plasmodium cynomolgi infection. Am J Trop Med Hyg 2008, 78:643-645.

35. van Duivenvoorde LM, van der Voorberg Wel A, Van der Werff NM, Braskamp G, Remarque EJ, Kondova I, Kocken CH, Thomas AW: Suppression of Plasmodium cynomolgi in rhesus macaques by coinfection with Babesia microti. Infect Immun 2010, 78:1032-1039.

36. Marley SE, Eberhard ML, Steurer FJ, Ellis WL, McGreevy PB, Ruebush TK 2nd: Evaluation of selected antiprotozoal drugs in the Babesia microti-hamster model. Antimicrob Agents Chemother 1997, 41:91-94.

37. Centers for Disease Control (CDC), USA, Weekly report: Clindamycin and quinine treatment for Babesia microti infections. MMWR Morb Mortal Wkly Rep 1983, 32:65-66. 72.

38. Lalloo DG, Shingadia D, Pasvol G, Chiodini PL, Whitty CJ, Beeching NJ, Hill DR, Warrell DA, Bannister BA: UK malaria treatment guidelines. J Infect 2007, 54:111-121.

39. Htut ZW: Artemisinin resistance in Plasmodium falciparum malaria. N Engl J Med 2009, 361:1807-1808. author reply 1808.

40. Phyo AP, Nkhoma S, Stepniewska K, Ashley EA, Nair S, McGready R, Ler Moo C, Al-Saai S, Dondorp AM, Lwin KM, et al: Emergence of artemisinin-resistant malaria on the western border of Thailand: a longitudinal study. Lancet 2012, 179:1960-1966.

41. Ansa GA, Walley JD, Siddiqi K, Wei X: Assessing the impact of TB/HIV services integration on TB treatment outcomes and their relevance in TB/HIV monitoring in Ghana. Infect Dis Poverty 2012, 1:13.

42. Zhou XN, Bergquist $R$, Tanner M: Elimination of tropical disease through surveillance and response. Infect Dis Poverty 2013, 2:1.

doi:10.1186/2049-9957-2-24

Cite this article as: Zhou et al:: Co-infections with Babesia microti and Plasmodium parasites along the China-Myanmar border. Infectious Diseases of poverty 2013 2:24

\section{Submit your next manuscript to BioMed Central and take full advantage of:}

- Convenient online submission

- Thorough peer review

- No space constraints or color figure charges

- Immediate publication on acceptance

- Inclusion in PubMed, CAS, Scopus and Google Scholar

- Research which is freely available for redistribution 